\title{
Review of the Research on Talent Ecological Environment Gu Ran ${ }^{1, a}$, Shang Hua ${ }^{2, b^{*}}$
}

${ }^{1}$ Faculty of Management and Economics, Dalian University of Technology, Dalian, Liaoning, China 2 Faculty of Management and Economics, Dalian University of Technology, Dalian, Liaoning, China aguran960617@163.com, bshanghua@dlut.edu.cn, *Shang Hua

Keywords: Talent, Ecological environment, Evaluate.

\begin{abstract}
With the increasingly globalization of economics, the competition for talents has become more intense worldwide in twenty-first century. The competition of talents is essentially the competition of talent eco-environment. At present, more and more research is focused on the evaluation of talent eco-environment. This paper combines different literature and finds some limitations. To solve these problems, this paper presents a method that introduces Bronfenbrenner's ecological systems theory into the research on talent eco-environment. Meanwhile, it also refers to some mature career development stage theories, considering the demand characteristics of talents who are in different stages of the career, so as to identify the factors that influence the evaluation of talent eco-environment. This paper not only provides a new perspective for the construction of the talent eco-environment, combines the research with the existing theories, but also puts forward considering the influence of time on talent selection creatively.
\end{abstract}

\section{人才生态环境研究述评 \\ 顾然 ${ }^{1, a}$ ，商华 ${ }^{2, b *}$ \\ ${ }^{1}$ 大连理工大学管理与经济学部, 大连, 辽宁, 中国 \\ ${ }^{2}$ 大连理工大学管理与经济学部, 大连, 辽宁, 中国 \\ auran960617@163.com, bshanghua@dlut.edu.}

*商华

关键词：人才；生态环境；评价

中文摘要. 随着经济全球化的深入, 21 世纪各国对人才的争夺变得愈加激烈。从本质上来说，人才的 竞争是人才生态环境的竞争。目前, 已有越来越多的研究聚焦在人才生态环境评价上。本文通过对文 献的梳理, 发现已有研究中的一些局限。针对这些问题, 本文提出可以将布朗芬布伦纳的生态系统理 论引入对人才生态环境的相关研究中, 同时参考一些成熟的职业发展阶段理论, 考虑处在不同职业生 涯发展阶段的人才的需求特征, 从而全方面的识别影响人才生态环境评价的因子。本文不仅为研究人 才生态环境评价提供了新的视角, 将现有的研究与相关理论结合, 而且创造性地提出了考虑时间因素 对人才选择的影响。

\section{1. 引言}

随着经济全球化的深入, 人才已成为 21 世纪各国竞相争夺的一种战略性资源。除了加强 对人才的培养外, 防止人才的流失也成为了各国发展建设中的重要问题。一个国家能否吸引 
世界优秀人才, 取决于这个国家能否为各类人才提供一个良好的成长和事业发展的生态环境。 从本质上来说, 国际各经济实体之间的人才竞争, 是人才生态环境的竞争 ${ }^{[1]}$ 。然而, 我国人才 流失问题一向严重, 优化我国的人才生态环境, 成为了增强我国核心竞争力的重要举措。目 前, 已有越来越多的学者加入到对人才生态环境的研究中。然而, 通过对文献的梳理, 我们 发现了现有研究中存在的一些局限。针对这些问题, 本文提出可以将布朗芬布伦纳的生态系 统理论引入人才生态环境的相关研究, 针对不同行业人才的特点, 同时参考施恩、格林豪斯 或萨柏等人较成熟的职业生涯发展阶段理论，考虑处在不同职业生涯发展阶段的人才的需求 特征, 全方面的识别影响人才生态环境评价的因子, 帮助企业甚至地区更好的优化人才生态 环境, 从而吸引和保留人才。

\section{2. 文献回顾}

\section{1 人才生态环境概念}

王顺较早地对人才环境进行研究, 他认为人才环境是人才赖以生存、得以发展的社会环 境和自然环境，人才环境的内容非常广泛，涉及政治、经济、文化、人文、科技、教育、地理

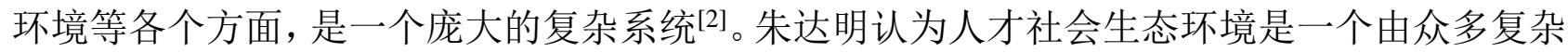
因素构成的社会系统, 是一个完整的、有内在规律的体系, 主要可分为社会物质环境和社会 人文环境 ${ }^{[3]}$ 。杜业艳认为人才生态环境是指能够确保人才生存发展、展示才华和实现其价值的 生活环境、工作环境、政治环境、文化环境和人文环境等的总和 ${ }^{[4]}$ 。刘瑞波、边志强认为人才 社会生态环境是指由诸多相互作用、相互制约的因素构成的完整的、有机的、可持续发展的 系统, 这个系统以人才为中心, 包含影响人才生存发展的经济、制度、物质、文化在内的各 种社会因素，各因素对人才的生存和发展具有直接或间接作用 ${ }^{[5]}$ 。

\section{2 人才生态环境评价指标体系构建}

在人才生态环境评价指标体系构建上, 我国学者主要从理论或实证两个方面来进行研究。 其中, 在理论研究层面, 大部分学者通过文献梳理, 进行了人才生态环境评价指标体系的构 建, 选取了人才生态环境的评价指标, 并探讨了良好的人才生态环境对于该地区经济发展的 意义等方面。邱安昌将东北人才生态环境分为人才的物质环境和社会人文环境。他认为, 人 才的物质环境和人才的人文环境还可以相应的转换为功能的 “硬环境” 和 “软环境”。“硬 环境” 泛指构成企业和人才生态环境的所有有形实体的硬件条件, “软环境” 是除硬环境外 所有对企业和人才有影响的其他因素, 即企业和人才生态环境中一切无形却具重大影响的因 素。“硬环境”与 “软环境” 相辅相成, 相互作用。并且, 决定企业和人才生存、发展的因素 逐渐由硬环境转变为软环境 ${ }^{[6]}$ 。黄梅、吴国蔚认为人才生态环境包括自然生态环境、社会生态 环境、规范生态环境等。人才的自然环境通常是指围绕人才生态主体的自然界, 包括非生物 环境和生物环境, 对人才的发展有着非常重要的影响。人才的社会生态环境是指自然生态环 境中独立出来的人才社会活动有关的社会影响因素，涵盖政治、经济、法律、科技等方面。 人才的规范生态环境是人类在群体生活中所形成和持有的态度, 风气, 观念。文化是人才生 态主要的规范环境 ${ }^{[7]}$ 。王亚男将区域战略性新兴产业人力资源生态系统分成了产业中人力资 源和产业内外部人力资源生态环境两部分，建立了基于生态位理论的区域性战略新兴产业人 力资源生态系统评价指标体系, 从生存力、竞争力和发展力三方面进行了评价。生存力和竞 争力反映了人力资源本身, 发展力反映了人力资源生态环境 ${ }^{[8]}$ 。

在实证研究层面, 大部分学者通过发放问卷, 采用德尔菲法, 实地访谈法等篮选评价体 系指标, 并采用数理统计法进行验证分析, 对实证研究的结果进行优化, 从而提出相应的人 才政策建议。李玉香认为, 人才环境只有被感知时才有意义, 她将研发人才环境感知分为人 才政策环境感知、事业环境感知、生活环境感知三部分，以深圳227家高新技术企业及其研发 人才为样本, 采用问卷发放和实地访谈等方法, 深入探讨了研发人才环境感知对研发人才工 
作绩效和工作嵌入的影响 ${ }^{[9]}$ 。韩俊从宏观生态环境和微观生态环境两大视角对科技创新人才 的生态环境进行了综合研究, 构建了科技创新人才生态环境与科技创新能力关系模型, 将科 技创新人才生态环境分解为创新精神、创新氛围和创新压力三大维度, 并以浙江省高校和企 业为样本进行了实证研究 ${ }^{[10]}$ 。王耿耿将企业专业技术人才成长需求分为三个层次,生存需求、 关系需求和自我发展需求。对直接影响企业专业技术人才成长的生态环境进行分析, 然后从 人才自我感知的角度, 探索影响专业技术人才成长的关键生态因子, 设计企业专业技术人才 成长生态环境的测评指标体系, 进而运用层次分析法和模糊综合评价法构建出企业专业技术 人才成长生态环境的测评模型 ${ }^{[11]}$ 。张立新从经济基础环境、成长激励环境、科技创新环境、 生活环境和区位环境 5 个维度笁选指标, 最终形成了包括恩格尔系数、高新技术企业数量等 30 个二级指标的评价体系, 并采用非整秩次加权秩和比评价法 (WRSR) 对山东省17地市进行了 评价, 诊断分析各地市科技人才生态环境存在的问题, 提出相应的优化思路和对策 ${ }^{[12]}$ 。商华、 惠善成、郑祥成以辽宁省区域城市为样本, 以2013年辽宁省统计年鉴中数据为依据, 通过引 入生态位理论研究城市人力资源生态系统, 从动态和静态两个方面表征辽宁省区域城市人力 资源生态系统的竞争关系 ${ }^{[13]}$ 。

\section{3. 结论与展望}

通过对文献的梳理, 我们可以发现: (1) 现有的研究大多聚焦在人才生态环境的评价上, 各学者在进行研究时, 都根据人才的不同特点, 建立了适合于不同人才的生态环境评价指标 体系。但是其评价指标的选取往往是采用主成分分析或因子分析、层次分析法、模糊评价法 等不同数理统计方法搭配使用的方式, 或基于对文献综合整理的定性分析而成, 运用已有成 熟的生态学理论方法进行研究的比较匮乏。（2）各学者在研究时忽略了人才所处的职业生涯 发展阶段对其的影响, 目前所建立的评价指标体系大多是静态指标体系, 没有引入时间进行 参照。（3）各学者建立的评价指标体系不够全面, 其对人才生态环境的研究很少将省市区等 宏、中观层面和企业内部的微观层面相结合, 构建成一个整体的系统, 通常都是进行单方面 的研究, 这使得人才生态环境的评价指标体系缺乏结构上的完整性。

生态学是研究生物个体与其环境相互作用的科学, 也是一种认识论和方法论 ${ }^{[6]}$ 。美国心理 学家布朗芬布伦纳于1979年较系统地将生态学的知识引入到人类行为的研究中, 提出了生态 系统理论。他认为发展的个体处在从直接环境到间接环境的几个环境系统的中间或嵌套于其 中, 包括微系统、中系统、外系统和宏系统, 这些系统与个体互相作用, 并影响着个体的发 展。这一理论强调了环境作为一个复杂的系统对人的发展的重大影响, 发展来自于人与环境 的相互作用，相互作用的过程设定了人的发展路线。

综合以上研究成果, 在未来的研究中, 我们可以尝试将布朗芬布伦纳的生态系统理论引 入到对人才生态环境的研究中, 构建基于生态系统理论的人才生态环境评价指标体系。同时, 也可结合施恩、格林豪斯或萨柏等人的职业生涯发展阶段理论, 考虑不同职业生涯发展阶段 的人才特征及其需求差异, 全面地识别影响人才生态环境评价的各个因素, 以此为我国人才 生态环境的优化和可持续发展提供科学合理的建议, 从而吸引和保留人才。

\section{致谢}

本文为辽宁省省社科规划基金项目《辽宁省人才生态环境评价研究》(L16BRK001)、大连 理工大学创新训练项目《战略性新兴产业人才生态环境研究》（2016101410217）的阶段性成 果之一。 


\section{References}

[1] Peng Jianfeng, WTO and improvement and optimization of human resources ecological environment in China, Human Resource Development of China, no.01, pp. 7-10, 2002.

[2] Wang Shun, Study on the synthetic evaluation indicator system of China's s city HR environment, China Soft Sciences, no.03, pp. 148-151, 2004.

[3] Zhu Daming, Strategy of building talent eco-environment, China Talent, no.06, pp. 57-59, 2004.

[4] Du Yeyan and Zhu Huajie, Ecological environment construction of human resources, The Science Education Article Collects, no.04, pp. 149-150, 2006.

[5] Liu Ruibo and Bian Zhiqiang, Study on evaluation system of science and technology talent social eco-environment, China Population, Resources And Environment, vol.24, pp. 133-139, 2014.

[6] Qiu Anchang and Wang Sujie, Northeast China's eco-environment for talent growth vs its evaluation, Dongjiang Journal, vol.25, pp. 84-89, 2008.

[7] Huang Mei and Wu Guowei, Study on the comprehensive evaluation system of talent ecological environment, Science and Technology Management Research, no.01, pp. 62-65, 2009.

[8] Wang Yanan, Wang Hongqi, and Li Yonghua, The design of evaluation index system for human resource ecosystem of regional strategic emerging industries, Statistics \& Decision, no.13, pp. 33-37, 2016.

[9] Li Yuxiang and Liu Jun, An empirical study of the impact of the perceived talent environment on performance and job embeddedness of the R\&D talents, Soft Science, vol.23, pp. 110-114, 2009.

[10] Han Jun, The research of macroscopical and microcosmic biological environment of the creative talents in science and technology, Hangzhou: Zhejiang University, pp. 23-28, 2011.

[11] Wang Genggeng, Research on ecological environment in which the enterprise technical talents' growth, Wuhan: Wuhan University of Technology, pp. 35-40, 2012.

[12] Zhang Lixin and Cui Lijie, Evaluation of eco-environment of science and technology talent in city region based on WRSR of non integer rank: A case study of 17 cities in Shandong Province, Science and Technology Management Research, no.2, pp. 83-87, 2016.

[13] Shang Hua, Hui Shancheng, and Zheng Xiangcheng, Research on the evaluation of cities human resource ecosystem in Liaoning based on the ecological niche model, Science Research Management, vol.35, pp. 156-162, 2014. 